Brit. J. industr. Med., 1958, 15, 250.

\title{
COUNTING ERRORS DUE TO OVERLAPPING PARTICLES IN THERMAL PRECIPITATOR SAMPLES
}

\author{
BY \\ S. A. ROACH \\ From the Pneumoconiosis Research Unit of the Medical Research Council, Llandough Hospital, near Cardiff
}

(RECEIVED FOR PUBLICATION APRIL 25, 1958)

A sample of airborne dust taken with the standard thermal precipitator consists of two deposits about $1 \mathrm{~cm}$. long and $1 \mathrm{~mm}$. wide obtained from either side of the heated wire (Green and Watson, 1935). These deposits are examined under a microscope and a count is made across each of them to estimate the number of particles collected.

The particles commonly have an irregular shape and some consist of several smaller particles clumped together. It is usually assumed that these clumps or " aggregates" of particles were present when the dust was airborne and they are therefore counted as single particles. During the sampling some of the particles are deposited so that they cover or overlap others already deposited, producing spurious aggregates. When examined under the microscope this type of aggregate cannot normally be distinguished from one which existed as such in the air and these aggregates are also counted as single particles. Consequently the airborne dust concentration is underestimated. In the many previous tests of the thermal precipitator the magnitude of this error does not seem to have been investigated.

If two instruments are operated close together and the volume of air sampled by one is greater than the volume of air sampled by the other, the deposits obtained with one instrument will be more crowded than the deposits obtained with the other and hence the airborne dust concentrations estimated from the two instruments will be different. Therefore it was thought that by comparing the results from pairs of samples taken in this way, with very different volumes, it would be possible to estimate the magnitude of the bias due to overcrowding that normally occurs.

As air is drawn through the thermal precipitator at a constant flow rate the volume of air sampled can be varied by altering sampling time.

\section{Experimental Procedure}

Two thermal precipitators were set up side by side underground in a colliery. Pairs of samples were then taken in which one of the instruments sampled for five times as long as the other. Each short-period sample was taken during the middle of the corresponding sample taken by the long-period instrument. One hundred and twenty-five such pairs of samples were taken in different places in the colliery so as to include a wide variety of dust concentrations.

All the samples were then counted using a $2 \mathrm{~mm}$. oil immersion objective, counting all visible particles. Then all the samples were re-counted using a $4 \mathrm{~mm}$. objective, restricting the count to those particles which were between 1 and 5 microns in diameter. In both cases any aggregate consisting of particles which overlapped one another was counted as one particle.

In this way 250 pairs of results were obtained which, in the absence of overlap errors, would be expected to agree after allowing for the different volumes of the samples.

To check that the two instruments gave the same result when sampling identically, 15 pairs of samples were taken in which both instruments sampled for 10 to 15 minutes and 15 pairs of samples were also taken in which both instruments sampled for two to three minutes.

Finally a detailed size analysis was done on a number of the less dense samples selected at random. The object of this was to compare the amount of overlapping found with the amount expected from theoretical considerations. This can be calculated from the size distribution by making certain simplifying assumptions about the way the dust deposits.

\section{Results}

A comparison of the mean counts of corresponding short- and long-period samples is given in Fig. 1. The short-period counts were grouped and averaged and the corresponding long-period means calculated. In the absence of overlap errors the counts from the long-period samples would be expected to be roughly five times those of the short-period samples. This is shown by the straight line. In fact, the counts from the long-period samples were only about three times the count from the short-period samples, suggesting that a considerable amount of overlapping occurred 

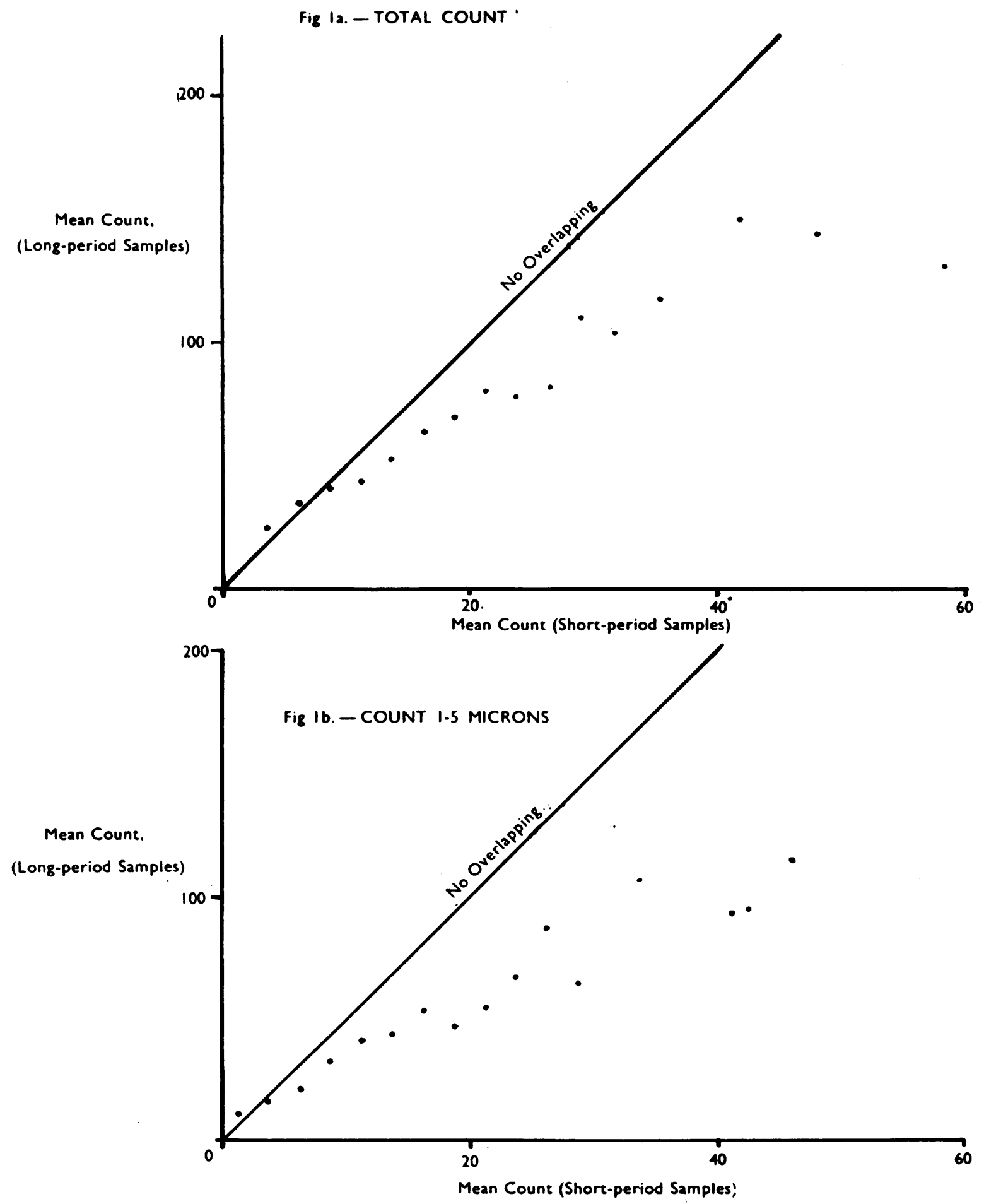

Fig. 1.-Comparison of short- and long-period samples (20 $\mu$ traverse). 
in the denser samples. This result was not unexpected since, when counting the densest samples, many long chains of overlapping particles could be seen.

A summary of the size distribution of the dust is given in Table 1. The mean projected area of the particles was 18.6 sq. microns.

TABLE 1

\begin{tabular}{c|c} 
SIZE DISTRIBUTION OF THE DUST \\
\begin{tabular}{c|c} 
Size Range \\
$(\mu)$
\end{tabular} & \% of Particles \\
\hline$<0 \cdot 5$ & 22 \\
$0 \cdot 5-1$ & 19 \\
$1-2$ & 21 \\
$2-3$ & 14 \\
$3-4$ & 9 \\
$4-5$ & 5 \\
$5-10$ & 7 \\
$10-20$ & 2 \\
$20-50$ & 1 \\
$<50$ & 0 \\
\hline Total & 100 \\
\hline
\end{tabular}

The agreement between the two instruments when sampling identically is shown in Table 2 . There is no suggestion from these results that one instrument was biased relative to the other.

TABLE 2

MEAN COUNTS FROM TWO THERMAL PRECIPITATORS SAMPLING IDENTICALLY

(20 micron traverse)

\begin{tabular}{|c|c|c|c|c|}
\hline $\begin{array}{c}\text { Period } \\
\text { Sampling }\end{array}$ & $\begin{array}{c}\text { Size } \\
\text { Range }\end{array}$ & T.P. & $\begin{array}{l}\text { Number of } \\
\text { Samples }\end{array}$ & $\begin{array}{l}\text { Mean } \\
\text { Count }\end{array}$ \\
\hline \multirow{4}{*}{ Long } & \multirow{2}{*}{ Total } & 1 & 15 & $60 \cdot 5$ \\
\hline & & 2 & 15 & $62 \cdot 9$ \\
\hline & \multirow{2}{*}{1 to $5 \mu$} & 1 & 15 & $33 \cdot 0$ \\
\hline & & 2 & 15 & 32.6 \\
\hline \multirow{4}{*}{ Short } & \multirow{2}{*}{ Total } & 1 & 15 & $41 \cdot 7$ \\
\hline & & 2 & 15 & 41.9 \\
\hline & \multirow{2}{*}{1 to $5 \mu$} & 1 & 15 & $17 \cdot 0$ \\
\hline & & 2 & 15 & $15 \cdot 8$ \\
\hline
\end{tabular}

Another possible source of bias was the extraneous contamination on the cover glasses. This might have affected the counts from the long- and short-period samples equally. To check this point counts were made on every cover glass at points a few millimetres from the deposits. These counts were made over a traverse length of $2 \mathrm{~mm}$., the maximum traverse length normally used when counting across a deposit. The counts were made using a $2 \mathrm{~mm}$. objective and all visible particles were counted. The mean number counted on the cover glasses carrying the short period samples was 1.3 particles per traverse 20 microns wide, and the mean number on the cover glasses carrying the long-period samples was 1.7 particles per traverse 20 microns wide. The contamination tended to be less on the cover glasses carrying the less dense samples. Thus the effect of contamination on the comparisons is likely to have been slight. In particular in the comparisons made at the higher densities these results suggest that the effect of the contamination was negligible.

\section{Analysis of the Results}

The analysis of the results is complicated by the fact that the counts from both the long-period samples and the short-period samples are affected by the overlap error, although to different extents.

The effect of overlapping is that the number of particles counted in unit area is less than the number actually deposited. With deposits of increasing density the number counted on each deposit would eventually cease to rise and would actually begin to fall as the gaps between the particles become fewer until finally all the particles overlap one another. That is, in the limit the number counted per traverse tends to zero. If, therefore, the number deposited in unit area could be plotted against the number counted, a curve of the form shown in Fig. 2 would be obtained. Initially the numter counted would be nearly equal to the number deposited, shown by the straight line. The number counted per traverse would rise to a maximum and then fall off, eventually tending to zero as the number deposited became very large.

Apart from these general properties the exact mathematical form of the relationship is not known. Curves relating the number counted $(y)$ to the number deposited $(x)$ were therefore fitted to the data by finding those which would account for the observed differences when using the equation,

where " $a$ " is a constant.

$$
y=x e^{-a x},
$$

This has the following advantages: (1) It has the general properties described above. (2) It is simple to fit to the data. (3) It is similar in form at low densities to the approximations suggested by Irwin, Armitage, and Davies (1949), Armitage (1949), and Mack (1956).

The ratio of the volumes used for the long- and short-period samples varied between 4.74 and 5.57 for different pairs of samples. The mean count from each long-period sample was multiplied by the ratio of the volumes of air actually sampled by the shortand long-period instruments, so as to make it comparable with the mean count from the short-period sample. A comparison of the results from the individual pairs of samples is given in Fig. 3 where the count from the short-period sample is plotted against the difference between it and the comparable count from the long-period sample. In the absence 


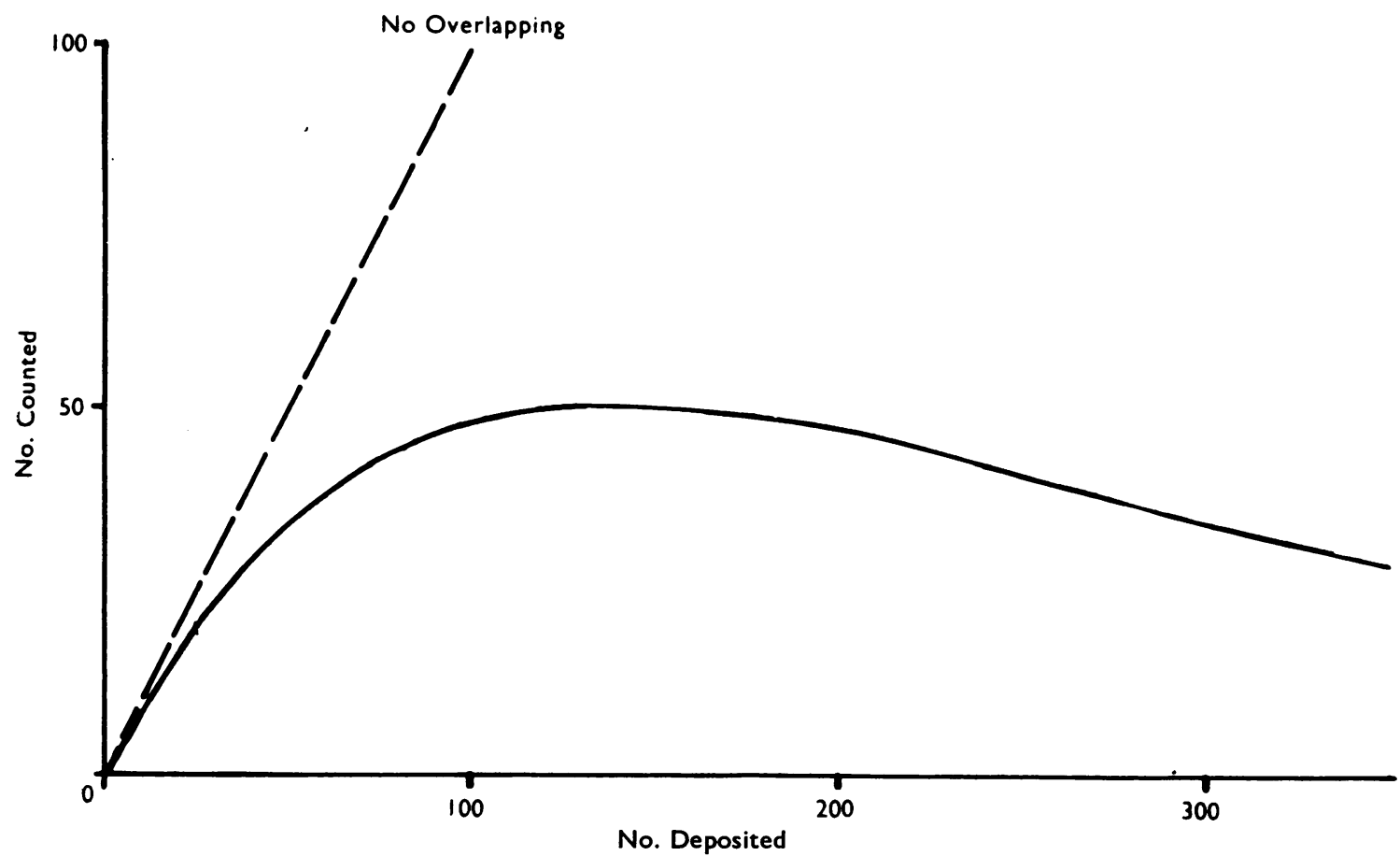

Fig. 2.-The number deposited in unit area and the corresponding number counted.

of overlapping we would expect the difference between the two counts to vary around zero. It can be seen that in practice the difference increases with the density of the samples, as we would expect if overlapping were taking place. Fig. 3 also shows that the fitted curves describe the relationship adequately.

The constant " $a$ " would be expected to depend on the size distribution of the airborne dust. The finer the airborne dust, the smaller will be the magnitude of " $a$ ", the more nearly will the number counted $(y)$ be equal to the number counted $(x)$ and the less will be the effect of overlapping on the number count.

From every pair of results it was possible to calculate a value for " $a$ ". It was expected that the counts obtained would be more variable than the size distribution, with the result that the variability of the values for " $a$ " obtained from the counts would fall off roughly as the inverse square of the count. This was found to be the case. The individual values of " $a$ ", for the total count and the count from 1 to 5 microns, were therefore weighted by the square of the count in deriving mean values.

Because of the high variability of the calculated value for " $a$ " at very low densities, mentioned above, and because these counts tend to be un- reliable owing to contamination and the difficulty of focusing on sparse deposits, in calculating the mean value for " $a$ " results were excluded where the total number counted on the short-period sample was less than 8 particles per traverse.

The mean value for " $a$ " was $\mathbf{0 . 0 0 2 6}$ for the total number count and $\mathbf{0 . 0 0 4 8}$ for the count 1-5 microns. The difference between the number deposited and the number counted can be estimated from these results. The number counted is less than the number deposited by an amount which increases with the density of the deposit. The corresponding correction to be added to the number counted is given in Fig. 4. The correction is expressed as a percentage of the number count.

\section{Discussion}

Green and Watson (1935) in their original description of the thermal precipitator recommended a density of 62 particles for a traverse 22.2 microns wide but thought counting was possible when the deposit was one-fifth or about 20 times as dense. They said: "At the higher limit aggregation and superimposition of particles may become serious obstacles to accurate counting." The Transvaal Chamber of Mines (1947), when referring to the counting of particles in a traverse 8.5 to 8.7 microns 


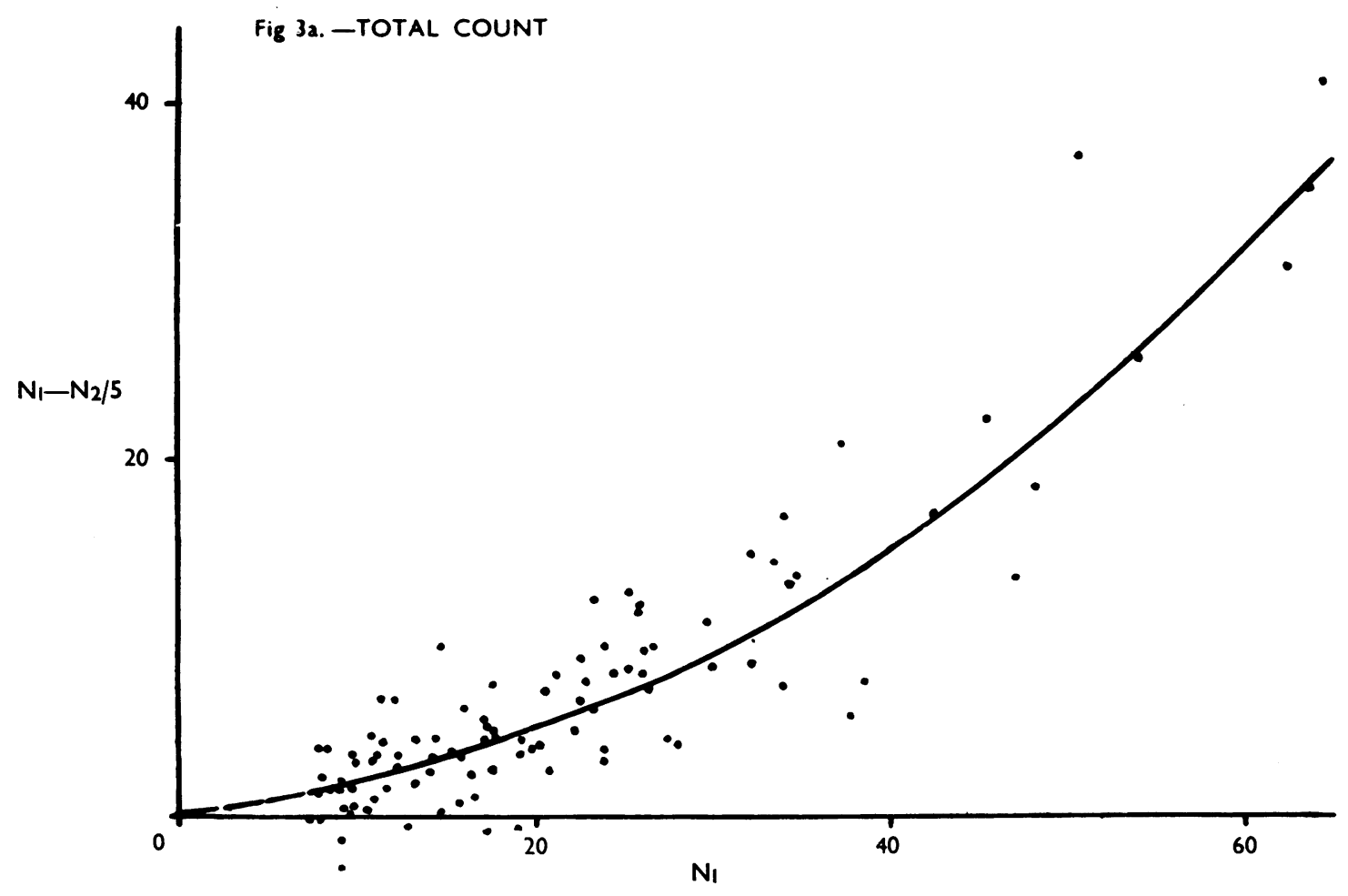

$N_{1}$

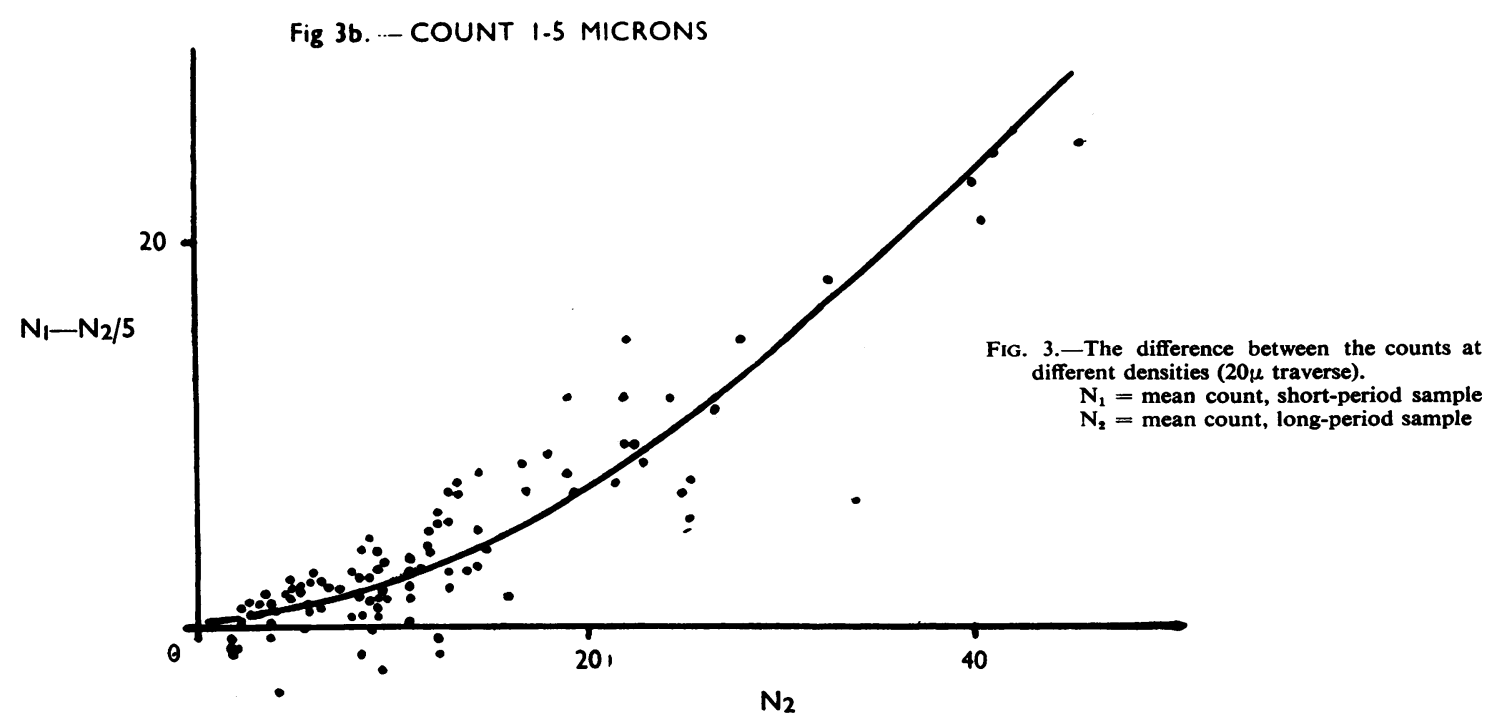




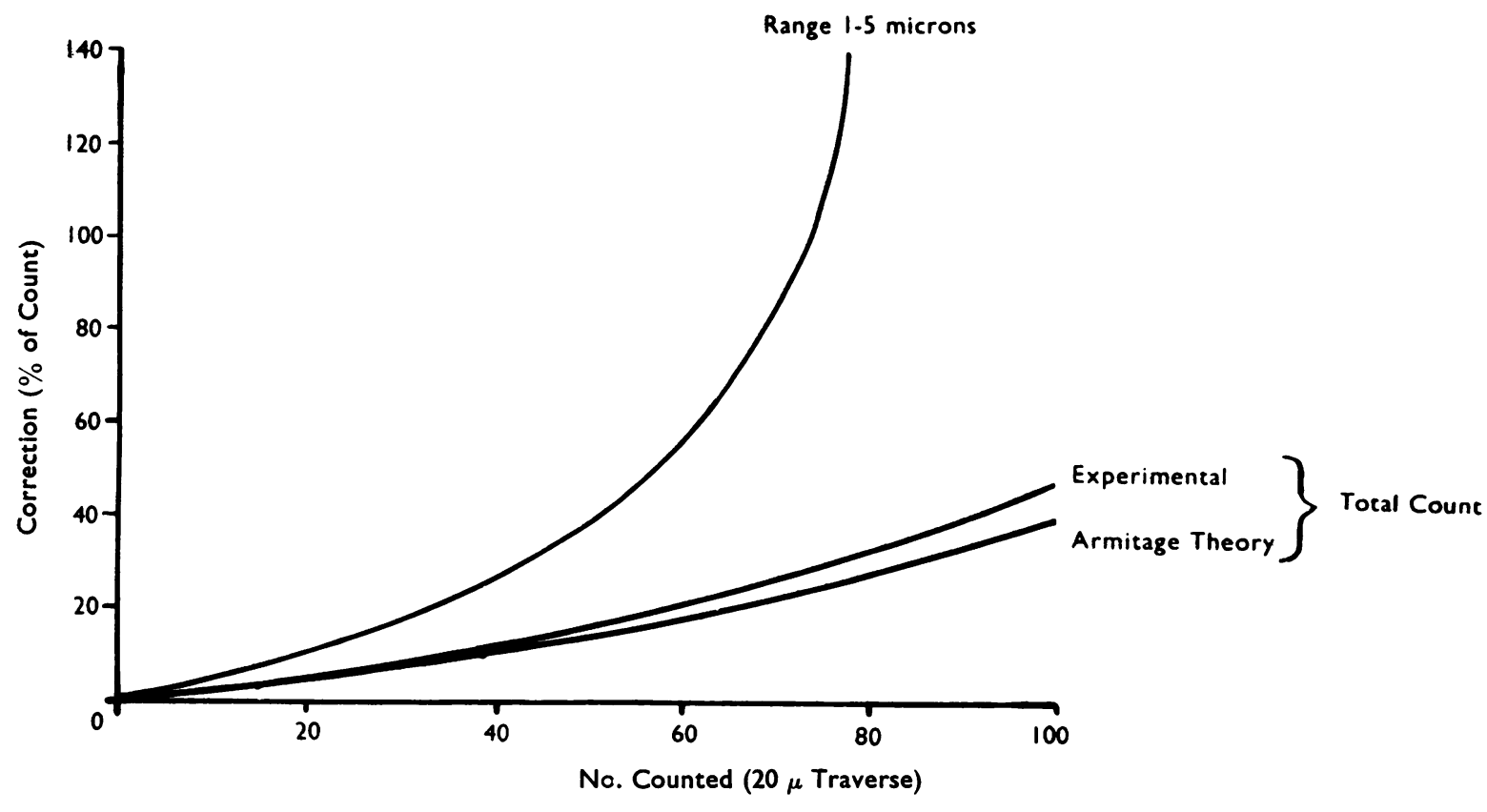

Fig. 4. - The correction to be added to the count.

wide, say, "If the record is too thick, crowding of the particles causes inaccuracy and counting becomes difficult and tiring. ..." and " the density should not be higher than about 300 particles per strip ". They say later, "A density of about 100 particles per strip is most satisfactory in practice and, so far as possible, sampling must be arranged with this object in view". They also point out that "It happens sometimes that the number of particles in a traverse is less than 50 particles. .."

Holdsworth, Henley Price, and Tomlinson (1954), when describing the usual procedure adopted in the National Coal Board, say, in regard to traverses 30 microns wide, "The density of the deposits is usually such that 100 to 150 particles are counted at each traverse for it is considered that less than 50 or more than 200 particles per traverse would introduce serious counting errors".

At the Pneumoconiosis Research Unit, we have been in the habit of using densities such that the total count lies between 50 and 100 particles for a traverse 40 microns wide and the count $1-5$ microns diameter lies between 25 and 50 particles for a traverse $\mathbf{4 0}$ microns wide, although occasional samples have occurred less than half and more than twice as dense as this.

In Table 3 these densities are referred to a count corresponding to a traverse 20 microns wide for comparison with the results described earlier and for reference to Figs. 3 and 4.
The results suggest that the sample densities normally used by this Unit are such that the true airborne dust concentrations are between 5 and $15 \%$ higher than those estimated from the counts. Green and Watson (1935) recommended higher sample densities than we use but it is possible that they had in mind much finer dusts than are normally found in coal-mines and the amount of overlapping would then be much less. The Transvaal Chamber of Mines (1947) recommended densities several times greater than we normally use although here again these densities may be tolerable if the dust is very fine. The sample densities used by the National Coal Board (Holdsworth et al., 1954) are between four and five times as great as those used by us, but they may make some attempt to count overlapping particles separately. However, Hicks (1952), when quoting the instructions given in a counting trial to

TABLE 3

RANGE OF DENSITY OF DEPOSIT USED BY DIFFERENT WORKERS STANDARDIZED FOR A TRAVERSE 20 MICRONS WIDE

\begin{tabular}{|c|c|c|c|}
\hline \multirow{2}{*}{ Size Range } & \multirow{2}{*}{ Worker } & \multicolumn{2}{|c|}{ Count per Traverse } \\
\hline & & Usual Level & Range \\
\hline All sizes & $\begin{array}{l}\text { P.R.U. } \\
\text { Green and Watson } \\
\text { S. Africa }\end{array}$ & $\begin{array}{l}25-50 \\
56 \\
233\end{array}$ & $\begin{array}{l}12-100 \\
11-1,118 \\
116-698\end{array}$ \\
\hline $1-5 \mu$ & $\begin{array}{l}\text { P.R.U. } \\
\text { Holdsworth et al. }\end{array}$ & $\begin{array}{l}12-25 \\
67-100\end{array}$ & $\begin{array}{l}6-50 \\
33-133\end{array}$ \\
\hline
\end{tabular}


compare the counts produced by the different Divisions of the National Coal Board, said:

“ The object of counting is to determine the number of airborne dust particles of a specified size in unit volume of air. These may be either behaving as single units or aggregates, that is, groups of adhering particles behaving as single entities. The criteria by which individuality for inclusion in the count should be judged are as follows: (i) that it falls within the specified size range; (ii) that at some stage of the focusing a clear margin of separation is visible between the particle and all its neighbours."

If this last rule is applied strictly, it seems unlikely that the counting is very different from that used in the experiment reported in this paper.

A number of workers have estimated theoretically the error due to overlapping which would be expected when particles are deposited at random on a sampling plate (see for example Armitage, 1949; Mack, 1954; 1956). However, they all make certain simplifying assumptions about the kind and size of aggregates produced which result in an underestimate of the error and are likely to be valid only when the number of particles overlapping is small. Moreover, the problem is complicated in the thermal precipitator for a number of reasons. Particles drawn into the thermal precipitator head are subjected to three main forces (Fig. 5); the force, $F$, due to the temperature gradient, the downward force of gravity $G$, and the viscous drag of the air, $R$. Consequently the particles, particularly the coarse ones, approach the depositing surface obliquely.

For a given number of particles deposited in unit area the risk of one of them being struck by an approaching particle is greater if the particle approaches obliquely than if it approaches vertically. All theoretical treatments of the problem have so far assumed that particles approach the surface vertically. This will lead to an underestimate of the actual amount of overlapping.

Another complication is that the theory assumes that the particles are equally likely to deposit anywhere on the depositing surface. In a thermal precipitator sample the density of the deposit varies over its width, being most dense near the centre; in addition coarse particles tend to deposit on one side and fine particles on the other. Also the place where a particle deposits may be influenced by particles already deposited exerting localized thermal or electrostatic effects.

Armitage (1949) derived an approximate expression for the effect of overlapping on the total number count, suitable for use when the amount of overlapping is small. He assumed the particles were laminae and were deposited at random on a sampling plate. The error in the total count predicted from these assumptions is shown by the bottom curve in
Fig. 4. This error is slightly less than that found experimentally with the thermal precipitator. This could be checked by comparing the thermal precipitator with a sedimentation cell. In the sedimentation cell there is no reason to doubt that the amount of overlapping would be the same as the amount predicted by Armitage.

\section{Conclusions}

When aggregates of particles were counted as single particles the error in the number count due to overlapping which occurred at particle densities commonly used was at least $10 \%$ when sampling

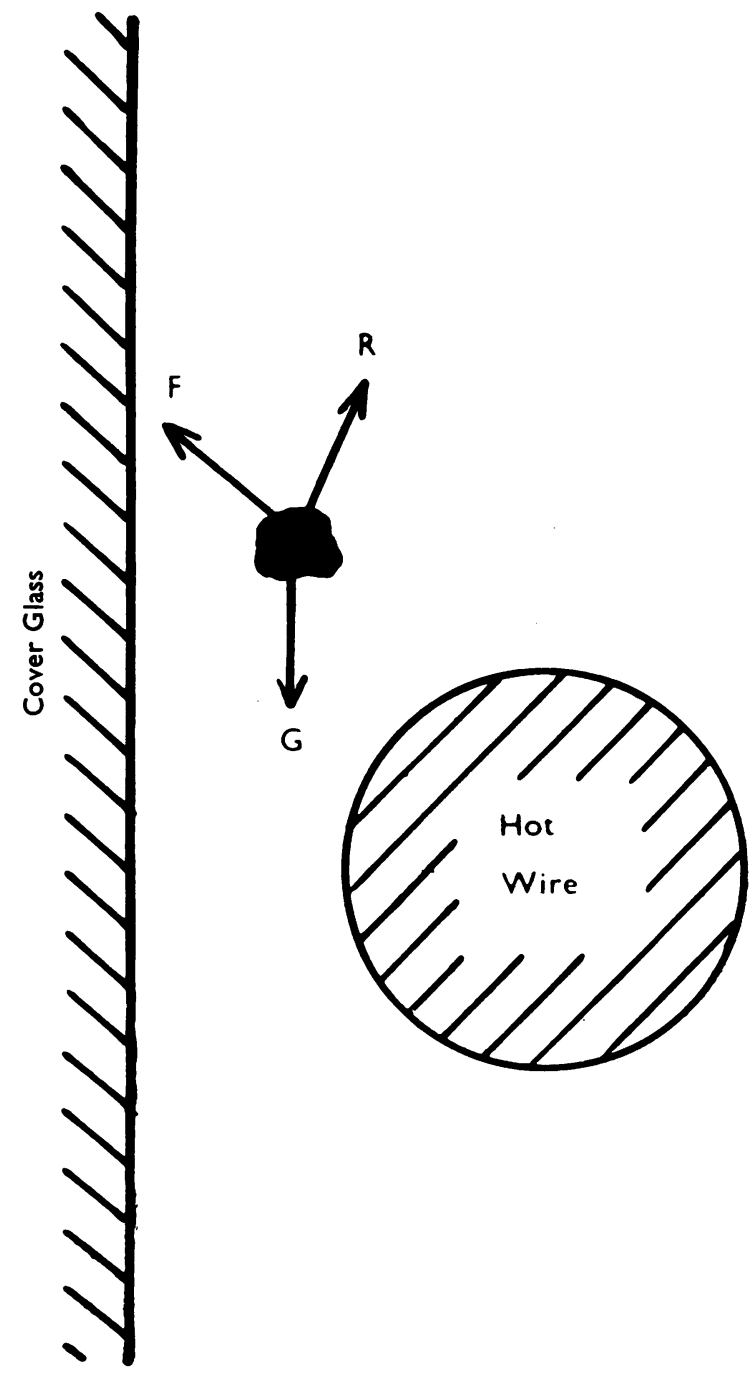

F1G. 5.-The forces on a particle in a thermal precipitator. 
the airborne dust in a coal-mine. This overlap error could be reduced by using lower densities, but against this must be weighed the error due to extraneous contamination, which would then become increasingly important. Assuming a maximum contamination of 3 particles per 20 micron traverse, then the particle density required to reduce this to say $5 \%$ of the count is 60 particles per traverse, a point where the error due to overlapping is already $20 \%$. Consequently, it does not seem to be possible in practice to avoid the difficulties introduced by overlapping particles when sampling airborne dust similar to that occurring in the mine where this investigation was made.

The overlap error would be expected to be less when sampling finer clouds. Also modifications of the standard instrument which have the effect of excluding large particles from the sample or spread the sample over a larger area are likely to be beneficial in this respect.

The effect of ignoring the overlap error is to compress the scale of dust concentration so that the average concentration is underestimated. Moreover, any changes in dustiness brought about by dust suppression are also underestimated.

In experiments to study the errors in thermal precipitator sampling it is customary to determine the repeatability of the results obtained by different instruments when sampling identically. Errors due to overlapping are not revealed in such experiments. Our results suggest that overlapping may be a serious source of error which should not be ignored.

Errors due to overlapping may produce misleading results when comparing different types of instruments. For example, Burdekin and Dawes (1956) compared the results of counts obtained using a standard thermal precipitator with those obtained using a thermal precipitator with an elutriator attachment to trap the larger particles before entering the instrument. Such a comparison would have only a limited value if high sample densities were used, as the effect of overlapping would be much less on the samples with a few large particles. Hamilton (1956) compared the results of counts obtained using a standard thermal precipitator with those obtained using a long-running thermal precipitator. The effect of overlapping on the counts obtained is likely to be different in the two instruments, as the long-running thermal precipitator has an elutriator attachment to trap large particles and in addition the depositing surface has a relatively large area.

\section{Summary}

In a thermal precipitator sample some particles overlap others and in consequence the concentration may be underestimated. The magnitude of this error has been estimated from a comparison of counts of pairs of samples taken in a colliery in which one sample of each pair was five times as dense as the other. This method of testing the instrument does not appear to have been reported previously.

It was concluded that the error in the number count due to overlapping which occurs at particle densities commonly used and recommended is large and cannot be ignored. This conclusion was supported by comparison with the error expected from theoretical considerations.

With the standard thermal precipitator the particle density required to reduce the overlap error to negligible proportions appears to be so low that its value as a standard reference instrument in coalmines is open to doubt and needs reinvestigation against an absolute standard.

Mr. P. D. Oldham of the Pneumoconiosis Research Unit suggested this experiment. The work was carried out by Messrs. B. P. Pryce, R. P. Pryce, and W. J. Davies, and the results were analysed by Dr. M. E. Wise. I would also like to thank Dr. J. C. Gilson, the Director of this Unit, for his many valuable suggestions in the preparation of this paper.

\section{REFERENCES}

Armitage, P. (1949). Biometrika, 36, 257. Burdekin, J. T., and Dawes, J. G. (1956). Brit. J. industr. Med., 13, 196.

Green, H. L., and Watson, H. H. (1935). Spec. Rep. Ser. med. Res. Coun.' (Lond.), No. 199.

Hamilton, R. J. (1956). J. sci. Instrum., 33, 395.

Hicks, D. (1952). The Sampling and Analysis of Airborne Dust for Control Purposes. Meeting of Experts on the Prevention and Suppression of Dust in Mining, Tunnelling and Quarrying. International Labour Office, Geneva.

International Labour Office, Geneva.
Holdsworth, J. F., Henley Price, F., and Tomlinson, R. C. (1954) Brit. J. appl. Phys., Suppl. 3, The Physics of Particle Size Analysis, p. 96.

Irwin, J. O., Armitage, P., and Davies, C. N. (1949). Nature (Lond.), 163,809

Mack, C. (1954). Proc. Camb. Phil. Soc., 50, 581.

(1956). Ibid., 52, 246. Transvaal Chamber of Mines (1947). Quality of Mine Air. Johannes-
burg. 